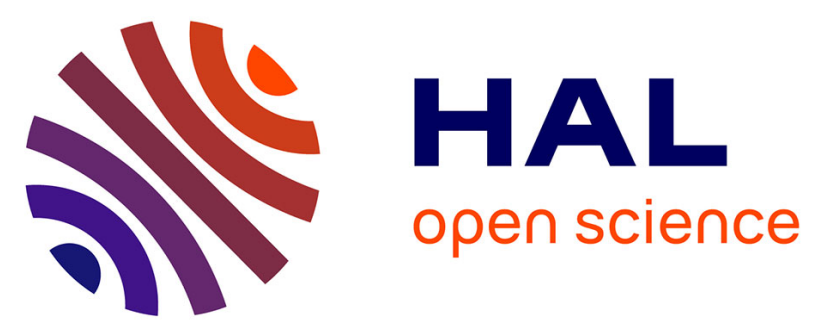

\title{
Faucon noir UAV project development of a set of tools for managing, visualizing and mosaicing centimetric UAV images
}

\author{
Antoine Gademer, Florent Mainfroy, Laurent Beaudoin, Loïca Avanthey, \\ Vincent Germain, Corentin Chéron, Sébastien Monat, Jean-Paul Rudant
}

\section{To cite this version:}

Antoine Gademer, Florent Mainfroy, Laurent Beaudoin, Loïca Avanthey, Vincent Germain, et al.. Faucon noir UAV project development of a set of tools for managing, visualizing and mosaicing centimetric UAV images. IEEE International Geoscience \& Remote Sensing Symposium, 2009, Barcelona, Spain. 10.1109/IGARSS.2009.5417833 . hal-01132115

\section{HAL Id: hal-01132115 \\ https://hal.science/hal-01132115}

Submitted on 16 Mar 2015

HAL is a multi-disciplinary open access archive for the deposit and dissemination of scientific research documents, whether they are published or not. The documents may come from teaching and research institutions in France or abroad, or from public or private research centers.
L'archive ouverte pluridisciplinaire $\mathbf{H A L}$, est destinée au dépôt et à la diffusion de documents scientifiques de niveau recherche, publiés ou non, émanant des établissements d'enseignement et de recherche français ou étrangers, des laboratoires publics ou privés. 


\title{
FAUCON NOIR UAV PROJECT DEVELOPMENT OF A SET OF TOOLS FOR MANAGING, VISUALIZING AND MOSAICING CENTIMETRIC UAV IMAGES
}

\author{
A. Gademer ${ }^{\dagger \S}$, F. Mainfroy ${ }^{\dagger}$, L. Beaudoin ${ }^{\dagger}$, L. Avanthey ${ }^{\dagger}$, V. Germain ${ }^{\dagger}$, C. Chéron ${ }^{\dagger}$, S. Monat and J.P. Rudant $^{\S}$ \\ ${ }^{\dagger}$ Pôle Acquisition et Traitements des Images et des Signaux (ATIS) \\ École Supérieure d'Informatique d'Électronique et d'Automatique (ESIEA), France, \\ Contact: beaudoin@esiea.fr, www.esiea-recherche.eu \\ $\S$ Université Paris-Est
}

\begin{abstract}
Eager for greater flexibility, and lesser costs, we are often tempted to acquire high-resolution data with home-made acquisition systems. However, this approach cannot benefit from usual tools to tackle problems such as image navigation within a large flow of very high resolution pictures, data completion and image mosaicing of small patches of the overflown area. This article presents the tools suite our laboratory has developed. In our case, we have developed homemade quad-rotor for low altitude imagery, equipped with up to three compact cameras on a stabilized platform. This configuration represents 2800 images at a ground resolution of 2 to 5 centimeters for each 20 minute flight. In order to take advantage of this flow of data, we needed a relevant set of tools for optimal acquisition and exploitation of the images.
\end{abstract}

Index Terms - UAV, high resolution imagery, flight-plan, intuitive database navigation, image mosaicing

\section{INTRODUCTION}

With the spreading of Geographic Information Systems and free visualization tools such as Google Earth ${ }^{\mathrm{TM}}$, more and more users tend to use satellite and aerial photography in their work to save time and reduce financial cost of their field study.

Nowadays, one can see growing need for very high resolution imagery in various fields such as crisis management (risk map, unreachable place exploration, damage assessment), biodiversity study (land-cover map, extended ground truth), radar images interpretation (roughness estimate) or even recreative cartography (virtual reality panorama).

From this point of view, UAVs (Unmanned Aerial Vehicles) are very interesting tools for low altitude imagery. Quickly deployable, they can fly when you need them, as often as you need without extracost. On the downside, the area covered are limited to a few hundred meters, trajectory is more sensitive to wind and thus less rectilinear and at this resolution the flow of data can grow huge rapidly. Therefore it is vital to develop a relevant set of tools to optimize the data acquisition and exploitation from the UAVs.

We will first present the Libellule Violette software which is an important part of our ground control station. This software applies optimization algorithm to the flight path to reduce the flight time and

This work was supported by ONERA and DGA in the Challenge Minidrones contest: http://minidrones.fr/ thus increase the coverage of the UAV. It also has a real-time completion detection algorithm which allows the pilot to know during the flight if there is no missing data and allows him to correct it before the landing, saving the cost of a backup flight.

Then we will present the Drone Eye software, our data exploitation library, which allows the end-users to navigate instinctively in the image database, just after the UAV landing, by an accurate indexation of the geolocalized images.

We will end this article with JIM, a powerful home-made opensource image mosaicer, that we have used to make planar mosaics of the scene.

\section{UAS}

Unmanned Aerial System acronym cover all the elements orbiting around the UAV : the quad-rotor itself and its control ground station, the payload and even the exploitation station, that allow the visualization of the data. It is the all-inclusive product the end-user will receive.

\subsection{UAV}

The UAVs history began in the early $\mathrm{XX}^{\text {th }}$ century where the military discovered that they needed an unmanned aircraft for two main missions : to be able to self-destruct on precise location or to be used as mobile target for the training of jet pilots. With the improvement of technology, UAVs are entrusted with more and more missions both military (intelligence, weapon, cargo, etc.) and civil (security, rescue, cartography, etc.). They come in all shapes and sizes, from automatized real scale plane to micro coaxial rotors UAV $[1,2]$.

In our case we have made the choice of a small scale quad-rotor. Indeed, this fixed structure with four fixed blade rotors has a lot of advantages, as it is simple from the mechanical point of view, sturdy and very maneuverable. Its degree of freedom comes from the differential speed of the pair of rotors, thus needing quite efficient control algorithms as it is intrinsically unstable. (cf. Fig 1)

The last technical hindrances for the democratization of civil UAV have been solved recently : higher energetic density with the Lithium-Polymer battery, higher efficiency with brushless motors and a global miniaturization of all the electronic components (internal sensors, computing chip, detection sensors, etc.) with the microelectromechanical systems (MEMS) and the surface mounted components (SMC). All of these reduce drastically the weight of the system and thus allow the building of small UAV, fitted for operational missions, at a reasonable cost. 


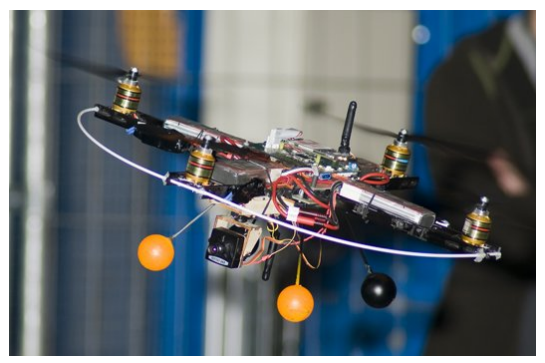

Fig. 1. The quad-rotor maneuverability is obtained by the differential speed of the rotors.

\subsection{Payload}

The payload corresponds to the weight of material the UAV can lift besides itself ; 1 kilogram, in our case, without limiting our maneuverability. Therefore the payload is also the material dedicated to the UAV's mission. Depending on our mission, it can be a photo camera, a video camera, a thermal camera, a passive or active sensor in all the frequency spectrums. It can be a single sensor or in group of sensors within reasonable size and weight.

[3] shows the interest of simultaneous multi-acquisition of high resolution images for environmental applications and for quick cartography in crisis situation. For situation assessment, operational users need information as much as possible ; they want recent, reliable images of their interest regions from several points of view to help the evaluation.

On the one hand, simultaneous acquisition reduces the number of flights needed to cover an area and thus increases the range of the UAV, it also permits to take a scene from different angles (cf. Fig 2), opening new data exploitation possibilities (intuitive navigation in the image database, stereoscopy, etc.). On the other hand the weight and size constraints become drastic and need more efforts on sensor integration.

As it can be seen in (cf. Fig 2), we use on-the-shelf photo camera in our system. Camera have the advantage of an autonomous storage capacity, all the processing of the image being done on board. They also have a big CCD captor, optics of professional quality, a mechanical shutter and an included stabilization system. However, these cameras are bigger and heavier than industrial CCD cameras and are made for human manipulation and thus are not easy to integrate in on-board systems.

Fortunately, after a slimming process and the development of an electronic card interface we are now able to control perfectly our three cameras and acquire simultaneous images from the sky.

\subsection{Geolocalization}

Relatively simple information is sufficient for the UAV stabilization, but the geolocalization of the data produced by the payload needs a more precise measurement of the trajectory and of the orientation of the UAV.

The problem is not simple as UAV have more complex trajectories than satellites or planes, they are more sensible to environment and their positions and heights can rapidly change due to a gust of wind. The main sensor on the UAV is the Inertial Measurement Unit (IMU) ; with accelerometers, gyroscopes and magnetometers it assesses the orientation of the UAV and thus allows to stabilize it. The orientation is also used to determine in which direction the payload's

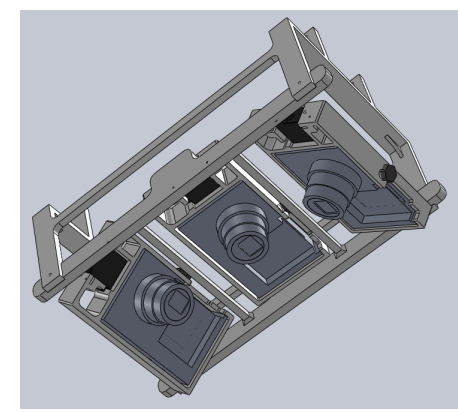

Fig. 2. The simultaneous multi-acquisition payload

cameras look. The position and altitude is given by the Global Positioning System (GPS) which can be accurate enough on flying systems. Another important information is the height above the ground, which is obtained by the data fusion of ultrasound, barometric and accelerometer systems.

To make things a little more complex, all these sensors measure physical property from which it derives the state of the system and thus present noisy and biased results. To make sure to have accurate knowledge of the true trajectory we must use complex filtering and data fusion [4].

All the tools we will present in the next section depend on the quality of this measurment and that is why one never stops working on improving it.

\section{DEDICATED TOOLS}

Acquiring images from UAV can be a bit challenging (limited flight time, huge flow of data produced, user craving for usable data, etc.) and therefore we need a relevant set of tools to help us during this process.

From our experience, obtain a fully operational UAS implies to place importance on the UAV and on the ground control station which allows the user to interface with the system, give it orders, check its status in real-time. The first two softwares that we will present are important part of the ground station : the mission programmer and the mission completion surveyor.

\subsection{Libellule Violette: Mission programmer}

The mission programmer is a tool which allows us to plan the UAVs missions. Among other things it offers to register, before take off, all the pieces of information we need in order to perform the mission in good conditions.

A flight-plan is composed of a sequence of geographic coordinates, which the UAV will follow during the mission. We thought that it would be easier to set them knowing approximately the scene we wanted to cover. Therefor we add a functionality to load any geolocalized image as a background map. The image can be loaded from the hard-drive or directly from Internet giving its centers geographical coordinates, a radius and a resolution. You can have the names of the streets and other items on this map or not according to your wishes. The map is directly downloaded and cached for later use.

Once loaded, the map is shown in background and you can move on it or change the zoom factor at any moment. 


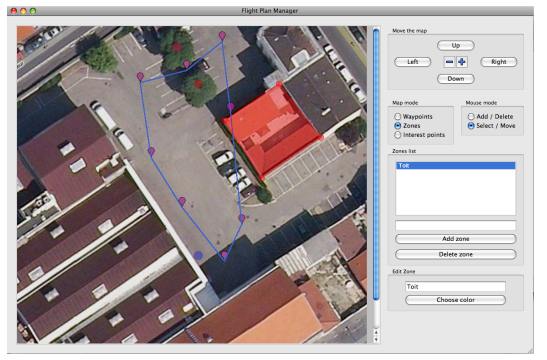

Fig. 3. The background map, with the flight-plan and the vector information.

On this image we can add manually some vector drawings, if we already possess some field information. The possibility to add areas and points of interest on the map can prove very useful during the planning and even during the flight as we will show in paragraph 3.2. For example it is important to note any uneven relief or obstacle. It can also be used to mark any change seen between the map and the reality as seen on the field. Every object can be customized with an identifier and a color so it is easy to track it down instantaneously on the map. (cf. Fig 3)

Once aware of the characteristics of the area, the user can plan the flight by adding, deleting and moving way-points. The program will calculate in real-time the optimized path to cover all the waypoints in the shortest time. A traveling salesman algorithm has been integrated for optimal calculation. In the future we hope to add other algorithm based on multiple criteria (distance, priority, sensor ground-print, etc.)

All the raster and vector informations can be saved in XML format to be reloaded later and to be use by the other programs, as the mission completion surveyor.

\subsection{Libellule Violette: Mission completion Surveyor}

The ground station gives in real-time the progress of the UAV on the flight-plan map to check his status and the correlation between the flight and the planned mission. We wanted to do more.

As cartographic UAV users, the most important thing for us is to avoid incomplete data. If we have two or more pictures of the same scene we can choose the best one but if we lack one, we are not able to give a correct assessment and that is not desirable. Most of the systems (satellite, aerial or UAVs) take a security margin : all the images cover a great percentage of the others, just in case. We wanted to optimize our flight and reduce this margin but how can we assure that we will not forget any important image ?

It is the job of the mission completion surveyor. This program, active during the flight use the data from the flight-plan programmer to show the progress of the UAV. But it takes advantage of all the information about the images we received in near real time from our UAV.

Firstly, as we have real-time retransmission of an on-board mini video camera, we add the functionality to edit during the flight all the vector information of the flight-plan. Therefore, it is possible, as in the mission programmer phase to add areas and points of interest, to tag them, to modify them, and so on. So for example, we may want to indicate that an area is flooded, or that there are rock slides and mass of fallen rocks at this place, that a detail requires ones interest here, that this area is marshy and this one forested, or whatever else you want to be highlighted on your map.
Secondly, we use the trajectory information paired with the time of acquisition of the pictures to calculate the approximate ground print of the pictures. Similar to the "fog-of-war" you can see in video games, all the places that have been seen by the UAV's payload are highlighted. All remaining areas are darkened, so the ground station user can see rapidly if there is any lack of data or not and possibly edit the flight plan as a result.

Finally, we made the data structure of these maps simple to be able to share with all the operational users on the ground, and that again in near real time. This map will allow them to get an overall view of all the data gathered and then they can act accordingly.

Now we can assure before the landing of the UAV that we have all the images we want, we will need to transfer and process them to be integrated in our image database. It is the role of our next tool, named Drone Eye.

\subsection{Drone Eye: Generating pertinent image database}

As seen in section 2.2, our small quad-rotor UAV can carry up to three compact cameras on a stabilized platform. From the operational point of view, that represents a total of 2800 images at a resolution of 2 to 5 centimeters for each 20 minute flight, which would be completely useless without powerful tools to index and visualize those pictures. We have developed an innovative solution to transform these raw images into highly valuable scenes.

The first step of our solution is to geolocalize the acquired data, using our embedded and GPS and Inertial System. We are thus able to compute the footprint of every picture and infer topological relationships between them. To ease the process, we use the WGS84 datum for GPS compliance (longitude, latitude, altitude coordinates), and a local tangent plane (north, east, up coordinates) which makes local navigation in our images database fast and easy.

The geographic metadata are then stored as separated XML files. Our first idea was to store these metadata into EXIF, but this data format does not provide a field for all the information we need, for example the attitude of the camera, which is obviously needed to reproject the pictures on the ground. Storing metadata in separated XML files, and not in the images files, has another benefit: we are dealing with very small text files and do not have to manipulate large binary images files when looking for a specific image. XML format also ensures temporal durability of those critical links between pictures and their geographic coordinates. These data are then stored in an XML Native Database (XND). The choice of using an XND was driven by the need of a fast access to metadata, in order to make navigation and visualisation seamless. This database scheme fully brings the benefits or XML trees, and only pertinent data is requested using the XQuery language. It is necessary make the distinction between XML-enabled databases and XML Native databases. The former are conventional databases able to parse and request XML text files, as $\mathrm{XND}$ are designed specifically for this task. XND are much faster though when dealing with XML files than conventional databases.

\subsection{Drone Eye: Visualization \& Navigation}

As seen in section 3.3, we have designed a way to access our images through geographic requests.

We developed a software solution to access our pictures in a natural way, through the use of Google Earth ${ }^{\mathrm{TM}}$.

Users navigate through the database with the help of their keyboard, using only a few keys to access desired pictures. Valid requests are "next image north from this one", "same location but with a 45 point of view", "same location but viewed from the south". 


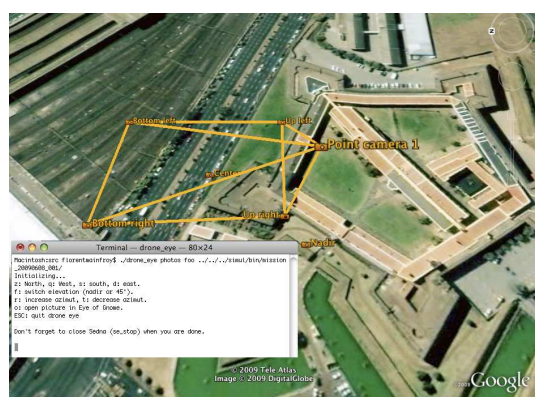

Fig. 4. Images can be requested with the keyboard and are immediatly displayed in Google Earth ${ }^{\mathrm{TM}}$.

Figure 4 shows Drone Eye usage : an image from the database is currently displayed, and the user can navigate through images around the area using the keyboard, as entered keys are intercepted by Drone Eye, which displays requested pictures.

Technically speaking, every adjacent image to the current one is requested, using XQuery language, from the XND. When the user accesses another image, it can be displayed very fast as we don't have to search the entire database.

Drone Eye is the glue between the XND, Google Earth ${ }^{\mathrm{TM}}$ and the user, which hides complex geographic projections and matrices mathematics, and lets the end-user concentrate on his needs: "show me the next image on the left", with a very intuitive navigation system.

\subsection{JIM: Open-source automatic mosaicker}

As we acquire multiple images from a very low altitude, we have multiples patches, each covering a very narrow area.

This approach gives centimetric resolution, but denies the user the possibility of getting the global picture.

We have developed our open-source solution to automatically generate mosaics from our images based on [5].

The first and critical step of our algorithm is to find repeatable interest points on acquired scenes. Different point detectors are suitable for this kind of application, but [6] recommend using Harris corner detector [7] for its high repeatability. To be more precise, we use an evolution of this detector described by [8]. We use Harris's [7] corner detector to ensure free software compliance. We then use Zero-mean Normalized Sum of Square Differences (ZNSSD) to match interest points and derive the most likely homography from these matches using RANdom SAmple Consensus (RANSAC) [9].

More matches are then found using guided matching based on the homography found previously, which makes the software able to refine the transformation between images.

If overlapping between two (or more) non consecutive images is detected, Jim is able to recompute only the necessary transformations using the $\mathrm{N}$-views graph described by Capel, taking advantage of Dijkstra algorithm [10] to minimize error propagation.

Transformed images are then mapped on a plane, using the previously computed homographies.

But image mosaicing would only be stitching without radiometric rearrangement. Jim is here helped by Enblend, another very efficient and open-source tool, which detects radiometric seams and moves separations between images in high frequencies areas, to make the transitions between images almost invisible.

Jim can be downloaded under GPL v3.0 on SourceForge.net.

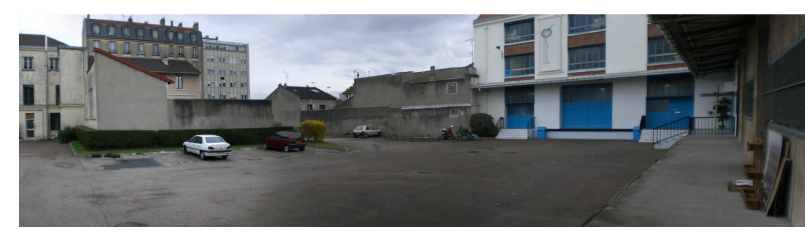

Fig. 5. A mosaic realized by JIM and Enblend using 6 12Mpx images.

\section{CONCLUSION}

To conclude we will say that the development of a set of tools to optimize acquisition and exploitation of data from UAV is very important. It is the missing link between the UAV makers and the operational users around the world.

We showed how promising UAV would be in the near future for the civil users and how important a precise geolocalization was. All ground station software allows the optimization of the flight to increase the range of the system. Exploitation software should give the operational users the ability to make a relevant use of this new type of data.

We will end, saying that we are currently running field test with end-users that will be the subject for a future publication.

\section{REFERENCES}

[1] J.R. Martnez de Dios, L. Merino, A. Ollero, L.M. Ribeiro, and X. Viegas, Multiple Heterogeneous Unmanned Aerial Vehicles, Springer Berlin / Heidelberg, 2007.

[2] K.P. Valavanis and M. Kontitsis, Advances in Unmanned Aerial Vehicles, Springer, 2007.

[3] A. Gademer, L. Beaudoin, S.Monat, C. Cheron, and J.P. Rudant, "Réalisation d'un drone pour des missions de télédétection THR environnementales à la demande," in XIième journées scientifiques du réseau télédétection de l'AUF, 2008.

[4] A.B. Chatfield, Fundamentals of high accuracy inertial navigation, AIAA, 1997.

[5] D. Capel, Image Mosaicing and Super-Resolution, SpringerVerlag, 2004.

[6] C. Bauckhage and C. Schmid, "Evaluation of keypoint detectors," Tech. Rep., INRIA, 1996.

[7] C. Harris and M. Stephens, "A combined corner and edge detector," in Proc. Fourth Alvey Vision Conference, 1988, pp. $147-151$.

[8] J. Shi and C. Tomasi, "Good features to track," in IEEE Conference on Computer Vision and Pattern Recognition (CVPR'94), Seattle, 1994.

[9] M.A. Fischler and R.C. Bolles, "Random sample consensus: a paradigm for model fitting with applications to image analysis and automated cartography," Commun. ACM, vol. 24, no. 6 , pp. 381-395, 1981.

[10] E. W. Dijkstra, "A note on two problems in connexion with graphs," Numerische Mathematik, vol. 1, no. 1, pp. 269-271, 1959. 